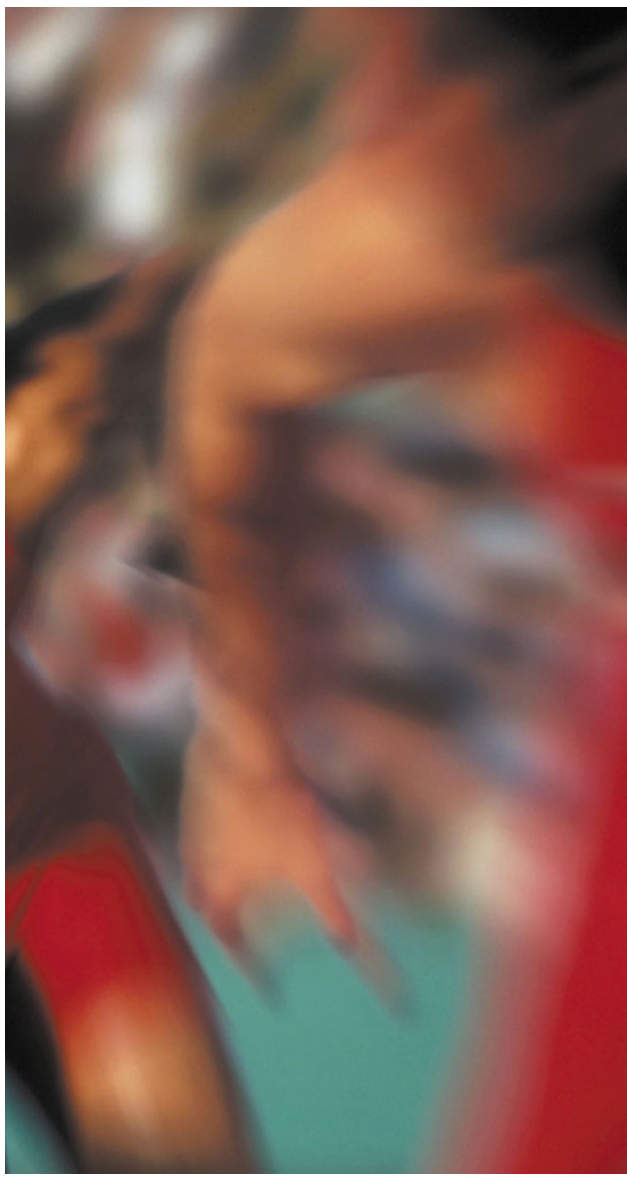

AIB1-overexpressing cancer cells and suggest new therapeutic avenues.

Cath Brooksbank

(2) References and links ORIGINAL RESEARCH PAPER Bai, J., Uehara, Y. \& Montell, D. Regulation of invasive cell behaviour by taiman, a Drosophila protein related AIB1, a steroi receptor coactivator amplifed breast cancer. Cell 103, 1047-1058 (2000)

So which signal is the strongest if a protein contains both a FCYENE signal and an ER retention signal? On the cell's factory floor, safety and quality control are of paramount importance, so the ER retention signal overrides the export signal, presumably to prevent the plasma membrane from being flooded with misfolded proteins.

Is FCYENE the only signal for export? Apparently not; Kir1.1 has an unrelated sequence at its $C$ terminus - VLSEVDETD - that also does the trick, and the vesicular stomatitis virus Gprotein has a DXE motif that is thought to speed its transit to the cell surface. No doubt these hint at more to come.

Cath Brooksbank

(2) References and links ORIGINAL RESEARCH PAPER Ma, $\mathrm{D}$. et al. Role of ER export signals in controlling surface potassium channel numbers. Science $\mathbf{2 9 1}$ 316-319 (2001)

H I G H L I G H T S

APOPTOSIS

\section{Into the groove}

One way to inhibit apoptosis is by blocking the activity of caspases, a job done by the inhibitor of apoptosis (IAP) proteins. Last year saw the identification of a mitochondrial protein called Smac/DIABLO, which has the opposite effect - it counters the inhibitory effects of IAPs by physically interacting with them. The structural basis of this interaction is discussed by two papers in Nature, and the results might have implications for the design of small molecules to treat cancer.

Liu and colleagues solved the solution structure of the BIR3 domain of one IAP (X-linked IAP; XIAP) complexed with a functionally active peptide derived from the amino terminus of Smac/DIABLO. Similarly, Wu and co-workers report the high-resolution crystal structure of Smac/DIABLO in a complex with the XIAP BIR3 domain.

The BIR3 domain is a potent inhibitor for caspase-9, and wild-type Smac/DIABLO is known to interact with both the BIR2 and BIR3 domains of XIAP. The amino-terminal residues of Smac/DIABLO are needed for its function, and both papers identify the first four amino acids (Ala-Val-Pro-Ile) as being criti$\mathrm{cal}$ - they recognize a surface groove on the BIR3 domain. This recognition specificity is achieved through a series of hydrogen bonding and van der Waals contacts between the BIR3 and Smac/DIABLO residues.

How, then, will this information aid in drug design? Some cancers overexpress IAPs, preventing apoptotic removal of the cancerous cells. Small molecules that mimic the effects of Smac/DIABLO could therefore be good potential drugs for killing these cells. And armed with the knowledge of the structure of the Smac/DIABLObinding groove, it should be possible to design high-affinity compounds that can slot into this groove.

Alison Mitchell

(0) References and links ORIGINAL RESEARCH PAPERS Liu, Z. et al. ORIGINAL RESEARCH PAPERS LIu, $Z$. et al. STructur basis for binding of STaCDIABLO to theXIAP BR3 domain. Nature 408, 1004-1008 (2000) | Wu, G. et al. Structural basis of IAP recognition by Smac/DIABLO. Nature $\mathbf{4 0 8}$ 1008-1012 (2000)

\section{IN BRIEF}

\section{MEMBRANE FUSION}

Sequential action of two GTPases to promote vacuole docking and fusion.

Eitzen, G. et al. EMBO J. 19, 6713-6720 (2000)

Vacuole fusion in Saccharomyces cerevisiae can be dissected into three steps: priming, docking and fusion. The Rab protein Ypt7p regulates vacuole fusion after the priming step - it binds in its GTP-bound form to the HOPS complex, which docks the membranes together. Ypt7p is then no longer needed for fusion to proceed. Surprisingly, fusion requires another, unknown GTPase that might regulate the calcium release necessary for fusion.

\section{CELL DEATH}

Wee1-regulated apoptosis mediated by the Crk adaptor protein in Xenopus egg extracts.

Smith, J. J. et al. J. Cell Biol. 151, 1391-1400 (2000)

The protein kinase Weel inhibits the cell-cycle kinase Cdc2, but Smith and colleagues now find a new function for it. They identified Weel in a screen for proteins that interact with the adaptor protein Crk, which is needed for apoptotic signalling in Xenopus egg extracts. Weel, like Crk, is needed for apoptosis in this system and addition of Wee1 markedly accelerates apoptosis. By contrast, other Cdc2 inhibitors have no effect on apoptosis in this system.

\section{DEVELOPMENT}

Wasp, the Drosophila Wiskott-Aldrich syndrome gene homologue, is required for cell fate decisions mediated by Notch signalling.

Ben-Yaacov et al. J. Cell Biol. 152, 1-136 (2001)

WASP, first identified because its mutation causes Wiskott-Aldrich syndrome, is involved in translating signals into cell movement. Ben-Yaacov and colleagues now identify a single WASP-like gene in Drosophila and find that cell-fate decisions are disrupted in WASP mutants: the phenotype is enhanced by intoducing a weak mutant of the Notch receptor into the WASP mutants, and suppressed when the Notch pathway is elevated, indicating that WASP might be involved in Notch-dependent cell-fate decisions.

\section{NEUROTRANSMITTER RELEASE}

Complexins regulate a late step in $\mathrm{Ca}^{2+}$-dependent neurotransmitter release.

Reim, K. et al. Cell 104, 71-81 (2001)

Complexins bind to SNARE complexes in the brain. This study establishes their function as positive regulators of neurotransmission, acting at or after the $\mathrm{Ca}^{2+}$-dependent step. Whereas complexin-I knockout mice show no phenotype, complexin-I and -II double deletion mutants die a few hours after birth. Spontaneous neurotransmitter release is normal in these mice, but they fail to respond to action potentials. Complexins are unlikely to be calcium sensors, but probably interact with the calcium sensor (which might or might not be synaptotagmin). 